\title{
NUMERICAL SIMULATIONS OF THE SOLAR ORBITER ANTENNA SYSTEM RPW ANT
}

\author{
M. Sampl*, H. O. Rucker*, T. H. Oswald*, D. Plettemeier ${ }^{\dagger}$, \\ M. Maksimovic ${ }^{\ddagger}$, W. Macher*
}

\begin{abstract}
The high-frequency electric sensors onboard Solar Orbiter are part of the radio and plasma wave experiment (RPW). The sensors consist of cylindrical antennas (ANT) mounted on three booms extruded from the central body of the spacecraft. Due to the parasitic effects of the conducting spacecraft body and solar panels the true antenna properties (effective axes and length, capacitances) do not coincide with their physical representations. The numerical analysis of the reception properties of these antennas is presented. In order to analyze the antenna system we applied a numerical method. The current distribution on the spacecraft body and the effective length vector was calculated, by solving the underlying field equations using electromagnetic code. In the applied method the spacecraft is modeled as a mesh-grid.
\end{abstract}

\section{Introduction}

Solar Orbiter is a future medium sized (M-class) spaceborne mission lead by ESA to investigate the Sun (planned launch in 2017). Flying a novel trajectory, with partial Sunspacecraft corotation, the mission is intended to investigate in-situ plasma properties of the near solar heliosphere. In addition it is going to observe and characterize Sun's magnetized atmosphere and polar regions.

The spacecraft payload consists of remote and in-situ sensors and instruments, covering a large scientific observation range. Within this instrument package Solar Orbiter carries a radio and plasma wave instrument (RPW) onboard, which is a $0 \mathrm{~Hz}$ to $30 \mathrm{MHz}$ electric sensor unit consisting of cylindrical antennas and the receiver hardware. These three antennas are mounted on booms extruded from the central body of the spacecraft, spreading the antennas from the spacecraft main body.

\footnotetext{
* Space Research Institute, Austrian Academy of Sciences, Schmiedlstrasse 6, A-8042 Graz, Austria

† Technical University Dresden, Chair in RF and Photonics, Georg-Schumann-Strasse 9, 01187 Dresden, Germany

‡LESIA, Observatoire de Paris, UMR CNRS 8109, 92195 Meudon, France
} 


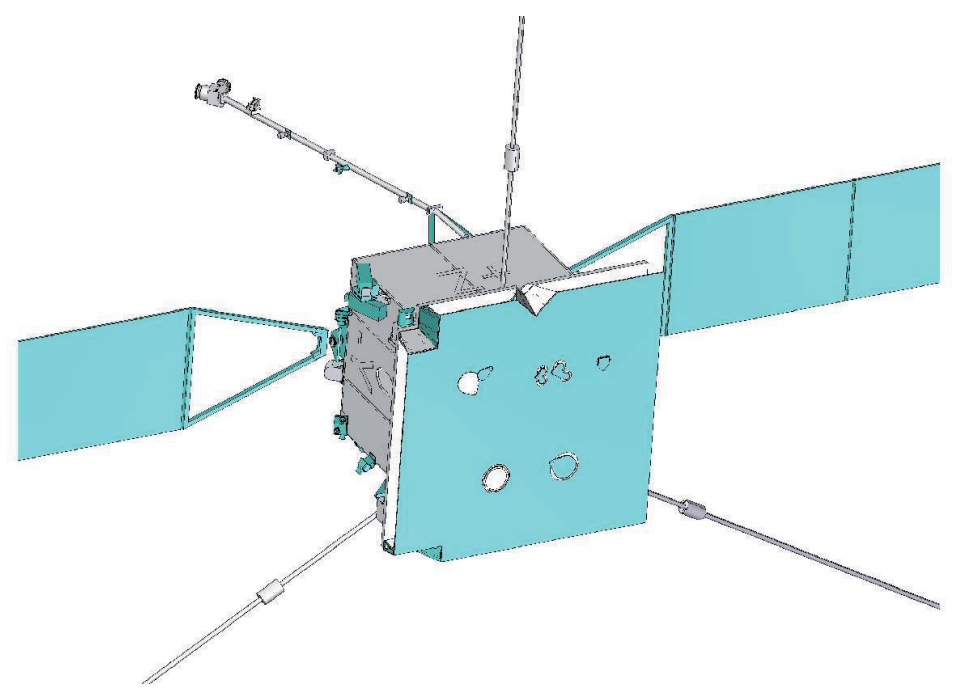

Figure 1: Preliminary CAD model of the spacecraft showing possible instrument and antenna configuration; the antenna system RPW ANT is represented by the boom antennas extruded from the central body

In this contribution the preparation of the Solar Orbiter spacecraft software model is outlined. A stable and good conditioned software model is the solid basis for future numerical simulations. Since the final spacecraft layout is not yet defined, this model can be used to investigate parasitic influences or antenna and object placement with regard to the radio and plasma wave experiment (RPW) onboard the spacecraft. In previous investigations, as in Fischer et al. [2001] or Oswald et al. [2009], the spacecraft body and antennas where modelled either as bodies with surface elements and antennas as wires or in total as wire-grids. An innovative step in software modelling was set by the Resonance project [Sampl, 2010, 2011] where the body and the antennas, for the first time, have been modelled as patches, greatly improving the accuracy of the acquired data. Notable is the fact that models with wire representations are sometimes resulting in low precision, as shown in the Resonance project [Sampl, 2010, 2011]. For example a wire is not an appropriate representation for a short cylindrical structure, solvers fail in obtaining a good result from such representations, due to an often implemented thin-wire approximation [Harrington, 1968]. Thus, the presented software model is fully comprised of surface elements as shown in section 2 .

In the present radio astronomy context the antennas properties are usually represented by the effective length vector $\mathbf{h}_{e}$ as defined by Sinclair [1950] and Macher [2005] for the quasi static range. Antenna properties at higher frequencies are often represented by radiation pattern and the antenna impedance. The RPW antenna properties of the current, preliminary spacecraft layout are presented in the final part 4 . 


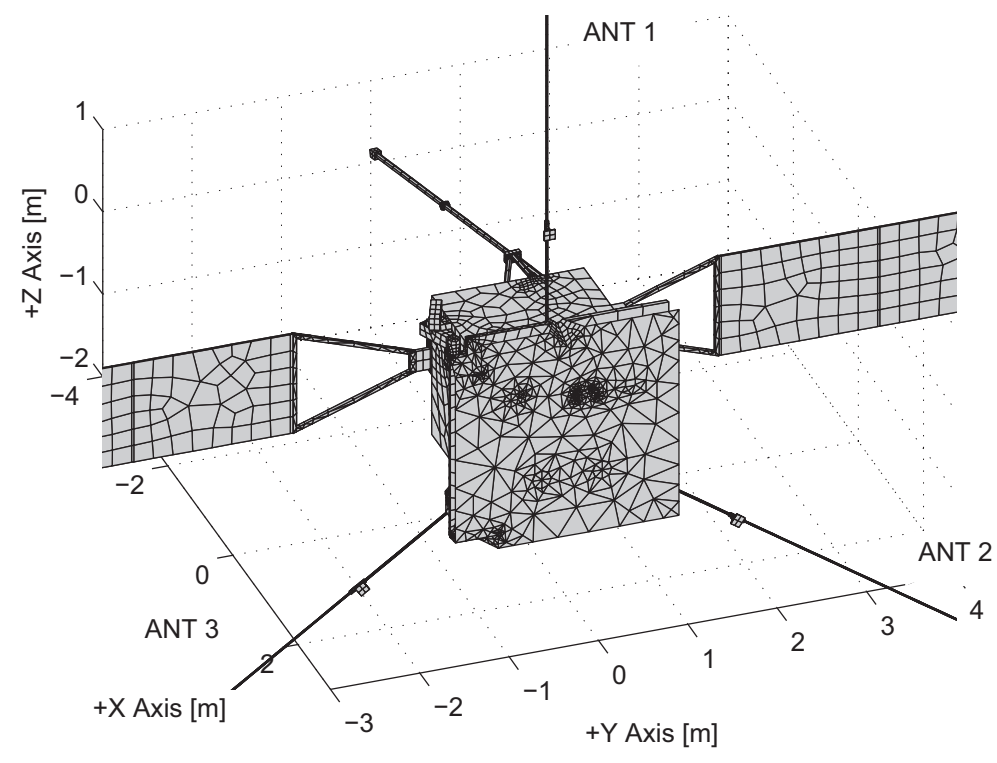

Figure 2: Spacecraft polygon mesh model consisting of approximately 8500 surface elements

\section{$2 \quad$ Spacecraft Modeling}

The basis of the software model for this investigation is CAD (Computer-aided design) data supplied by the RPW principal investigator and team in May 2010. Figure 1 shows a snapshot of the original data file, containing the spacecraft body, solar panels, preliminary instruments etc.

Since numerical calculations do not gain in precision using a model with the number of details exceeding a certain amount, it was appropriate to reduce the number of fine details in the drawing in the process of model authoring. In addition to that, commonly available electric field solver (method of moments) are not able to calculate models with a very high grade of fine details. The applied solver would either produce questionable results or hang-up during execution, often as a result of ill conditioned matrices.

The CAD file has been edited to the point where the balance of details and calculation time seemed to be in a healthy relation. Several parts of the spacecraft, including instruments and other details, had to be either removed or replaced by a simplified representations. The RPW ANT antennas have been completely replaced with self designed parts, in that step a preamplifier box outlined in the documentation was also added. The preliminary specification of the instrument and the antennas can be found in Maksimovic [2009].

The RPW monopole antennas (the other half of the dipole is represented by the spacecraft) 
are mounted on an antenna boom and extruded from the main body. The length of this separation boom was chosen for ANT1 as $1.2 \mathrm{~m}$, and for ANT2 and ANT3 as $1.5 \mathrm{~m}$. The antennas themselves are set at a length of $5 \mathrm{~m}$ and a diameter of $20 \mathrm{~mm}$ (cf. Maksimovic [2009]). The mentioned preamplifier box was added at the antenna foot point, to assess possible implications of such an object on the antenna impedances. Figure 3 pictures the preamplifier box from close up.

Finally the CAD model was converted into a polygon mesh with approximately 8500 surface elements (faces) and imported to matlabß.), as shown in Fig. 2, for calculation and analysis.

\section{Numerical Simulations}

The next step was to calculate the surface currents on the spacecraft and antennas. To achieve this, for each one of the antennas in the patch-grid model, said antenna was excited with 1 Volt and the solver CONCEPT-II ${ }^{1}$ was used to calculate the underlying electric field integral equation (EFIE). This takes approximately 6 hours on an average office computer per frequency step.

In our case the surface is assumed being a perfect conductor, then the EFIE [Macher, 2005] can be written as

$$
-\mathbf{n} \times \mathbf{E}^{\mathbf{i}}=\mathbf{n} \times \frac{1}{4 \pi} \int_{S}\left[\mathbf{J}_{S}\left(\mathbf{r}^{\prime}\right)\left(\frac{1}{j \omega \varepsilon} \nabla^{\prime} \nabla^{\prime}-j \omega \mu\right)\right] G\left(\mathbf{r}, \mathbf{r}^{\prime}\right) d S^{\prime}
$$

where $\mathbf{J}_{S}$ is the surface current of the patch-grid model, $S^{\prime}$ the applicable surface, $G\left(\mathbf{r}, \mathbf{r}^{\prime}\right)$ the scalar Green's function and $\mathbf{E}^{\mathbf{i}}$ an arbitrary excitation function.

Typical current distributions can be seen in Figure 3. Areas around the excitation zone have a high current density, where the color scale marks areas with high current density red, blending via green to blue for lower densities.

\section{Results}

Knowledge of the surface currents allows the calculation of the effective length and axes for each antenna, for the quasi static range:

$$
\mathbf{h}_{e}=\frac{1}{I_{a}} \int \mathbf{J}\left(\mathbf{r}^{\prime}\right) e^{j \mathbf{k} \cdot \mathbf{r}^{\prime}} d v^{\prime}
$$

\footnotetext{
${ }^{1}$ CONCEPT (COde for the Numerical Computation of Electromagnetic Processes for thin wire and thin shell structures) is developed by the Institute of Electromagnetic Theory at the Technische Universität Hamburg-Harburg
} 

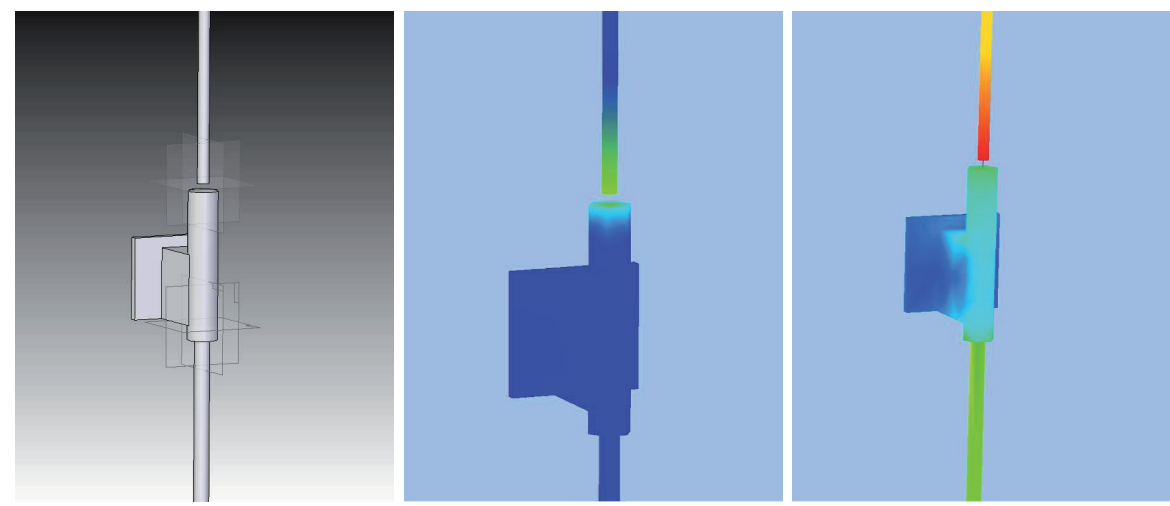

Figure 3: Current distribution $\left(\mathbf{J}_{S}\right)$ of the antenna feed zone; left to right shows: CAD snapshot, $\mathbf{J}_{S}$ at $300 \mathrm{kHz}$ and $\mathbf{J}_{S}$ at $15.2 \mathrm{MHz}$; the color scheme runs from red (high values) to blue (low values)

Table 1: Mechanical antenna vectors $\left(\mathbf{h}^{m}\right)$ of the Solar Orbiter antennas ANT1-ANT3, compared with their effective length vectors $\mathbf{h}_{e}\left(h_{e}^{o}, \phi^{o}, \theta^{o}\right)$ for open ports.

\begin{tabular}{ccccccc}
\hline & $\begin{array}{c}h^{m} \\
{[\mathrm{~m}]}\end{array}$ & $\begin{array}{c}\phi^{m} \\
{[\mathrm{deg}]}\end{array}$ & $\begin{array}{c}\theta^{m} \\
{[\mathrm{deg}]}\end{array}$ & $\begin{array}{c}h_{e}^{o} \\
{[\mathrm{~m}]}\end{array}$ & $\begin{array}{c}\phi^{o} \\
{[\mathrm{deg}]}\end{array}$ & $\begin{array}{c}\theta^{o} \\
{[\mathrm{deg}]}\end{array}$ \\
\hline ANT1 & 5.0 & 90.0 & 0.0 & 4.55 & 77.6 & 0.07 \\
ANT2 & 5.0 & 90.0 & -125.0 & 4.7 & 77.7 & -130.6 \\
ANT3 & 5.0 & 90.0 & 125.0 & 4.7 & 77.7 & 130.6 \\
\hline
\end{tabular}

Table 2: Capacitance $\mathbf{C}_{m}$ matrix for the $R P W$ antenna system, quasi static range

\begin{tabular}{lccc}
\hline Antenna & $\begin{array}{c}\text { ANT1 } \\
{[\mathrm{pF}]}\end{array}$ & $\begin{array}{c}\text { ANT2 } \\
{[\mathrm{pF}]}\end{array}$ & $\begin{array}{c}\text { ANT3 } \\
{[\mathrm{pF}]}\end{array}$ \\
\hline ANT1 & 44.99 & -2.91 & -2.91 \\
ANT2 & -2.91 & 45.35 & -3.05 \\
ANT3 & -2.91 & -3.05 & 45.36 \\
\hline
\end{tabular}

The integral in (2) has to be taken over a volume containing all surface currents, preferable the spacecraft volume. For the present setup, the effective length vectors of the RPW ANT antennas 1-3 are shown in Table 1 and Figure 4. The effective axes are tilted away from the mechanical rods by several degrees. The effective lengths are shortened, but not to a large extent. The holding booms separating the antenna from the spacecraft body add significant length to the overall result. The length of the holding boom for ANT1 is $1.2 \mathrm{~m}$ and $1.5 \mathrm{~m}$ for the antennas ANT2 \& ANT3. The difference in length was chosen to see the direct impact on the respective $\mathbf{h}_{e}$ as a hint for the holding boom selection on the actual spacecraft. 


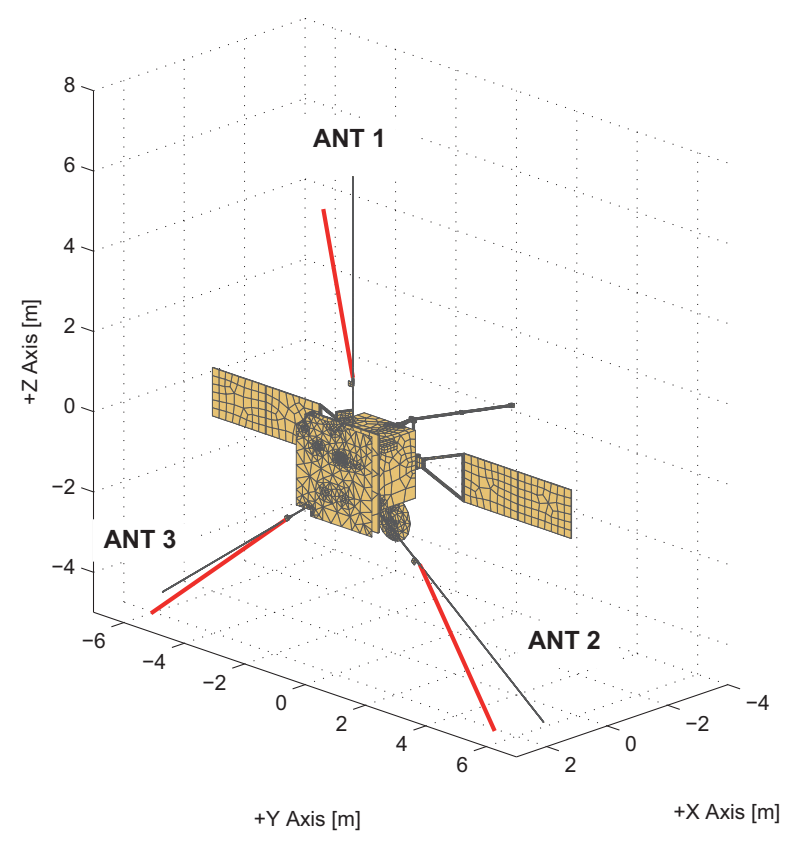

Figure 4: Solar Orbiter software model showing the effective length vectors $\mathbf{h}_{e}$ (red lines) of the $R P W$ antenna system.
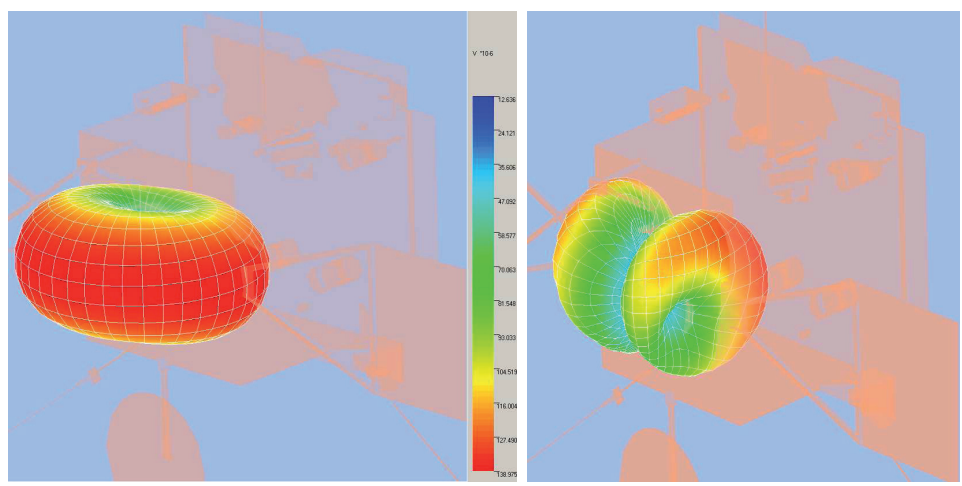

Figure 5: Radiation pattern of antenna ANT1; the left figure shows the situation at $400 \mathrm{kHz}$ and the right one at the second resonance frequency of $15.2 \mathrm{MHz}$; the color scheme runs from red (high E-field magnitude) to blue (low values). Coordinate axes of the pattern and spacecraft model are identical to those in Fig. 4. 
In addition to that, the calculation of the surface currents allows to predict the antenna impedances, which are dominantly capacitive for boom antennas, as shown in Table 2. Making a quick estimation using equation (3) [Jackson, 1962] shows that the acquired capacitance are in a valid range.

$$
C=\frac{2 \pi \varepsilon_{0} \cdot l_{A}}{\ln \left(\frac{2 \cdot l_{A}}{d}\right)-1}=50.5 \mathrm{pF}
$$

In equation (3) $l_{A}$ denotes the length of the antenna and $d$ the diameter.

At frequencies exceeding 1-2 MHz, beyond the quasi static range, the effective length vector is not appropriate anymore to describe the antenna parameters. The imaginary part of the effective length vector becomes significant, and it gets increasingly dependent on frequency and on the wave incidence direction. It is more useful to use a pattern to represent the antenna properties. In the course of the investigation, higher frequencies where calculated to find the relevant resonance points of the antennas. From previous investigations [Rucker et al., 2010] it is known that the second resonance is located between $14 \mathrm{MHz}$ and $18 \mathrm{MHz}$. This investigation is interesting, as the observation range of the RPW instrument will include areas beyond $20 \mathrm{MHz}$. The frequency range between $400 \mathrm{kHz}$ and $14 \mathrm{MHz}$, which obviously contains the first resonance frequency has not been calculated yet. Figure 5 shows two typical pattern of ANT1, for quasi static case at $400 \mathrm{kHz}$, where the pattern still has the typical donut shape and at $15.2 \mathrm{MHz}$ where the pattern is already heavily distorted and the donut is visibly split into two parts.

\section{Conclusions}

In this contribution a new software model and first results, regarding the radio and plasma waves experiment (RPW) onboard the upcoming Solar Orbiter spacecraft, are presented. In the course of the contribution the preliminary spacecraft design is used to shape a software model for electromagnetic field calculations. The presented model is comprised as a polygon face mesh, resulting in high precision data and appropriate ratio of precision and calculation time. First calculations present the effective length vectors and the capacitance matrix for the quasi static range, as well as patterns and surface current distributions for higher frequencies.

\section{References}

Fischer, G., W. Macher, H. O. Rucker, H. P. Ladreiter, D. F. Vogl, and the Cassini RPWS Team, Wire-grid modeling of Cassini spacecraft for the determination of effective antenna length vectors of the RPWS antennas, in Planetary Radio Emissions $V$, edited by H. O. Rucker, M. L. Kaiser, and Y. Leblanc, Austrian Academy of Sciences Press, Vienna, 347-356, 2001. 
Harrington, R.F., Field Computation by Moment Methods, Robert E. Krieger Publ. Company Inc., Malabar-Florida, 1st ed. 1968, reprint 1985.

Jackson, J. D., Classical Electrodynamics, John Wiley \& Sons, New York and London, 1962 .

Macher, W., Transfer matrix description of multi-port antennas and its application to the mars express/marsis radar, Ph.D. thesis, University of Technology, Graz, 2005.

Maksimovic, M., Specifications for the Antenna system (ANT) on the Solar Orbiter RPW experiment. SO-LES-SY-002, Version: 2, 11/09/2009, Observatoire de PARIS, 2009.

Oswald, T.H., W. Macher, H. O. Rucker, G. Fischer, U. Taubenschuss, J.-L. Bougeret, A. Lecacheux, M.L. Kaiser, and K. Goetz, Various methods of calibration of the STEREO/WAVES antennas, Adv. Space Res., 43, 355-364, 2009.

Rucker H.O., M. Sampl, M. Panchenko, T. H. Oswald, D. Plettemeier, M. Maksimovic, and W. Macher, Implications of antenna system calibration on spacecraft design and radia data analysis, Planetary Radio Emissions VII, Austrian Academy of Sciences Press, Vienna, this issue, 475-485, 2011.

Sampl, M., Experimental and numerical antenna calibration in the Resonance mission, Master thesis, University of Graz, 2010.

Sampl, M., Fundamentals of numerical analysis: spaceborne radio astronomy antennas. e \& i Elektrotechnik und Informationstechnik, vol. 128, 289-296, doi:10.1007/s00502011-0018-4, 2011.

Sinclair, G., The transmission and reception of elliptically polarized waves, Proceedings of the IRE, 38-2, 148-151, 1950. 